\title{
Theoretical analysis of electron-hole alignment in InAs-GaAs quantum dots
}

\author{
J. A. Barker and E. P. O'Reilly \\ Department of Physics, University of Surrey, Guildford, Surrey GU2 5XH, United Kingdom
}

(Received 19 November 1999; revised manuscript received 25 February 2000)

\begin{abstract}
We present a theoretical analysis of the mean electron and hole positions in self-assembled InAs-GaAs quantum-dot structures. Because of the asymmetric dot shape, the electron center of mass should be displaced with respect to the hole center of mass in such dots, giving rise to a built-in dipole moment. Theoretical calculations on ideal pyramidal dots predict the electron to be localized above the hole, contrary to the results of recent Stark-effect spectroscopy. We use an efficient plane-wave envelope-function technique to determine the ground-state electronic structure of a range of dot models. In this technique, the Hamiltonian matrix elements due to all components of the potential are determined using simple analytical expressions. We demonstrate that the experimental data are consistent with a truncated dot shape and graded composition profile, with indium aggregation at the top surface of the dot.
\end{abstract}

\section{INTRODUCTION}

There is considerable interest in the study of selforganized InAs-GaAs quantum-dot (QD) structures. These are interesting both from a fundamental physics perspective, and also because of potential device applications, particularly in the field of optoelectronics. ${ }^{1}$ Despite this, surprisingly little is known about their detailed atomic and electronic structure, including, for instance, the form of the ground-state electron and hole wave functions. Initial structural studies of uncapped Stranski-Krastinow (SK) dots suggested a pyramidal shape, ${ }^{2}$ and several groups therefore conducted theoretical investigations into the structure of ideal pyramidal dots. ${ }^{2-7}$ More recent structural investigations demonstrated that both the dot shape and size can vary with growth conditions. In addition to the ideal pyramidal shape, more recent work has also provided evidence for "truncated" pyramids, ${ }^{8}$ and for lens-shaped dots, ${ }^{9}$ as well as showing that the indium composition can also vary through the dot. ${ }^{10,11}$

Figures in most theoretical papers on ideal pyramidal dots show the presence of a permanent dipole in the dot, due to the large built-in strain fields which localize the highest energy hole state near the bottom of the pyramid, so that it sits underneath the lowest-energy electron state in the dot. Because of the built-in dipole, QD structures should exhibit an asymmetric Stark shift in the presence of an applied electric field. As an electric field $F$ is applied, the transition energy $E_{T R}$ between the ground-state electron and hole levels will vary quadratically as $E_{T R}(F)=E_{T R}(0)-\alpha F-\beta F^{2}$, where $E_{T R}(0)$ is the zero-field transition energy, $\alpha$ depends on the built-in dipole, and $\beta$ is a measure of the polarizability of the electron and hole wave functions. Asymmetry in experimental measurements of the Stark shift have indeed revealed a built-in dipole both in InAs-GaAs, ${ }^{12}$ and also in $\mathrm{Al}_{y} \mathrm{In}_{1-y} \mathrm{As}-\mathrm{Al}_{x} \mathrm{Ga}_{1-x} \mathrm{As}$ quantum dots ${ }^{13}$ but, surprisingly, the direction of the dipole is opposite to that predicted from the theoretical calculations, with the hole center of mass above the electron center of mass in both cases.

Here we present a theoretical investigation of the factors influencing the sign and magnitude of the built-in dipole in realistic QD structures. We use a carefully chosen one-band effective-mass model to investigate how the dipole $\alpha$ and the polarizability $\beta$ vary with dot shape, height, width, composition, and composition gradient. Most structural investigations suggest that SK dots have a broad base, tapering inwards toward their top, and also have a large base-to-height ratio. ${ }^{8,9,12,14,15}$ Here we consider ideal and truncated pyramids, and show that if one assumes constant composition in any such structure, then the built-in strain fields and large heavy-hole mass along the growth direction will lead to the hole ground state sitting near the dot base, below the electron, giving the opposite dipole to that observed experimentally. We must therefore modify this simple picture. Because the hole mass along the growth direction is considerably larger than the electron mass, the hole tends to sit in the region with the deepest potential while the electron is more widely spread through the structure. In order for the hole to sit above the electron as observed experimentally, the deepest potential must occur at the top of the dot. This can be achieved by increasing the indium composition from the base to the top of the dot, consistent with the conclusions of recent grazing-incidence diffraction measurements on uncapped InAs-GaAs QD's. ${ }^{10}$ We show that there exist a range of graded composition, tapered dot shapes that can give the correct sign and magnitude for $\alpha$. We also show that the magnitude of the polarization term $\beta$ is determined predominantly by the vertical height of the dot. The calculated values of $\beta$ are in good agreement with the experimentally determined values when the dot height is assumed to be comparable to that determined from TEM measurements of equivalent uncapped dots. ${ }^{12}$ Our calculations, combined with Stark effect measurements, provide useful information and sensible constraints on allowed models of QD's, consistent with a range of other experimental data.

The calculations were undertaken using separate one-band Hamiltonian equations for the electrons and holes, the details of which are described in Sec. II. We include anisotropy of the hole mass, and allow for a variation in the parameters and potential distribution due to built-in strain and compositional variation. We show that the wave functions and confinement energies calculated here for the ground-state electron and 
hole levels are in good agreement with data presented by other groups who used more sophisticated methods, such as eight-band k·P (Ref. 3) and empirical pseudopotential ${ }^{4}$ techniques. Although such techniques are necessary for the correct understanding of excited states in the dots, the linear and quadratic contributions to the Stark effect are determined primarily by the spatial distribution of the ground-state wave functions and by their potential profiles, each of which is well described here. The results of our calculations are presented in Sec. III. We first show that the results for conventional pyramidal dots are in good agreement with previous work, and reconfirm that the location of the hole below the electron in such a structure gives the wrong sign for the dipole. We then consider truncation of the pyramid, showing how the magnitude of the built-in dipole decreases with increasing truncation, but always remains positive so long as the dot tapers inwards toward the top. We explain this feature by using the calculated biaxial strain distribution within the dot, which has the effect of making the effective width of the top of the dot smaller for holes than for electrons, thereby maintaining the dipole. The inclusion of a wetting layer for $\mathrm{In}_{x} \mathrm{Ga}_{1-x} \mathrm{As} / \mathrm{GaAs}$ dots tends to make the dipole even larger, by acting to enhance the hole-trapping potential at the dot base while having relatively little effect on the groundstate electron. We then consider the case of a dot whose composition increases from base to top, considering both cuboids and truncated pyramids. For both cases this can lead to a localization of the hole above the electron, as observed experimentally. Looking at the polarizability $\beta$, we show that this is a strong function of the dot height but is relatively unaffected by the base dimension, and so may be used to estimate the vertical dimension of the dots. Finally, we carry out a brief error analysis, investigating how variations in key parameters affect the overall results. We find that changing the band-offset ratio or elastic constants used modifies the absolute values obtained for $E_{T R}(0), \alpha$ and $\beta$, but does not qualitatively affect the overall results. We omit the Coulomb interaction in our calculations but argue, based on analytical expressions presented as the Appendix, that this omission should also not qualitatively affect the overall results. We summarize our conclusions in Sec. IV.

\section{METHOD}

The calculations were undertaken assuming a threedimensional superlattice of dots, with superlattice unit cell size $2 L_{x} \times 2 L_{y} \times 2 L_{z}$. Schrödinger's equation for the system was solved using a plane-wave envelope-function technique. The normalized envelope function wave functions $\Psi_{n}(\mathbf{r})$ are determined using a Fourier series expansion

$$
\Psi_{n}(\mathbf{r})=\sum_{\mathbf{k}} \frac{A_{\mathbf{k}}^{n}}{\sqrt{8 L_{x} L_{y} L_{z}}} \exp (i \mathbf{k} \cdot \mathbf{r}),
$$

where $\mathbf{k}=\pi\left(m / L_{x}, n / L_{y}, p / L_{z}\right)$ and $\mathbf{r}=(x, y, z)$. We chose $(|m|,|n|,|p|) \leqslant(5,5,8)$ to ensure convergence, and chose the separation between neighboring dots to be equal to the dot dimension in order to minimize cross talk, while also ensuring efficient convergence of the Fourier series. We first de- scribe here the general method, and then discuss the particular form of the strained one-band Hamiltonian equations used.

Using the plane-wave basis, the energy levels and eigenfunctions were found by solving the large Hamiltonian matrix equation:

$$
\begin{gathered}
\sum_{\mathbf{k}} \bar{H}_{\mathbf{k}, \mathbf{k}^{\prime}} A_{\mathbf{k}}^{n}=E_{n} \sum_{\mathbf{k}} A_{\mathbf{k}}^{n}, \\
\bar{H}_{\mathbf{k}, \mathbf{k}^{\prime}}=\int_{\mathbf{r}} \frac{d \mathbf{r}}{8 L_{x} L_{y} L_{z}} \exp \left(-i \mathbf{k}^{\prime} \cdot \mathbf{r}\right) \hat{H}(\mathbf{r})[\exp (i \mathbf{k} \cdot \mathbf{r})] .
\end{gathered}
$$

In order to set up the Hamiltonian matrix of Eq. (2.1), we must first evaluate the matrix elements $\bar{H}_{\mathbf{k}, \mathbf{k}^{\prime}}$ linking planewave basis states of wave vectors $\mathbf{k}$ and $\mathbf{k}^{\prime}$. The lattice constant of the quantum dot material $\left(\mathrm{In}_{x} \mathrm{Ga}_{1-x} \mathrm{As}\right)$ differs from that of the surrounding matrix (GaAs) so that there will be a non-uniform strain distribution throughout the dot and the matrix. Both the electron and hole Hamiltonian equations contain terms depending on this local strain distribution. The calculation of the spatial strain distribution in a QD structure requires the solution of a three-dimensional problem in elasticity theory, for a generally nontrivial quantum dot shape. This is often achieved by using finite-difference or atomistic techniques, ${ }^{2,6,16}$ which require considerable computational effort. An analytical method was recently presented to calculate the Fourier transform of the strain distribution, ${ }^{17}$ which we use here to directly determine the strain-dependent terms in the Hamiltonian matrix. We assume for simplicity that the dot and matrix elastic constants are equal and isotropic, with the values used given in Table I.

We define the characteristic function of the $\operatorname{dot} \chi(\mathbf{r})$ to be the local indium composition in the supercell ( 1 for InAs, 0 for GaAs), and take the lattice mismatch of the dot $\varepsilon_{0}$ to be $6.7 \%$ for InAs in GaAs. It can be shown for an isotropic elastic medium that the Fourier transform of the real-space strain tensor component $\varepsilon_{i j}(\mathbf{r})$ is given by $\left(\mathbf{k}^{\prime \prime} \neq 0\right),{ }^{17}$

$$
\tilde{\varepsilon}_{i j}\left(\mathbf{k}^{\prime \prime}\right)=\chi\left(\mathbf{k}^{\prime \prime}\right) \varepsilon_{0}\left[\delta_{i j}-\left(\frac{C_{11}+2 C_{12}}{C_{11}}\right) \frac{k_{i}^{\prime \prime} k_{j}^{\prime \prime}}{\left|\mathbf{k}^{\prime \prime}\right|^{2}}\right],
$$

where $\mathbf{k}^{\prime \prime}=\mathbf{k}-\mathbf{k}^{\prime}, \chi\left(\mathbf{k}^{\prime \prime}\right)$ is the Fourier transform of the dot characteristic function, and $C_{11}$ and $C_{12}$ are the elastic constants. We wish to consider dots with a graded truncated pyramidal geometry. We define the truncation factor $f$ as the fraction removed from the top of the pyramid to give the truncated pyramid. A truncated pyramid of height $2 b_{z}$ and truncation factor $f$ is therefore the bottom part of a full pyramid of height $2 b_{z} /(1-f)$. We have derived that when such a truncated pyramid is centered at the origin of the unit cell, with base $2 b_{x}, 2 b_{y}$, truncated height $2 b_{z}$, and graded between an indium fraction of $f_{1}$ at the base, and $f_{2}$ at the (truncated) apex, the characteristic function $\chi\left(\mathbf{k}^{\prime \prime}\right)$ is given by 
TABLE I. Parameters used in this work.

\begin{tabular}{lcc}
\hline \hline & InAs & GaAs \\
\hline$m_{\text {exp }}^{*}(0 \mathrm{~K})^{\mathrm{a}}$ & 0.023 & 0.067 \\
$\gamma_{1}^{\mathrm{b}}$ & 19.67 & 6.85 \\
$\gamma_{2}{ }^{\mathrm{b}}$ & 8.37 & 2.10 \\
$a_{c}{ }^{\mathrm{c}}$ & -5.08 & -7.17 \\
$a_{v}{ }^{\mathrm{c}}$ & 1.00 & 1.16 \\
$e_{14} / \varepsilon_{r} \times 10^{-3} \mathrm{C} \mathrm{m}^{-2 \mathrm{~d}}$ & -2.97 & -12.49 \\
$E_{p}(\mathrm{eV})^{\mathrm{b}}$ & 22.2 & 25.7 \\
$\Delta_{0}(\mathrm{eV})^{\mathrm{b}}$ & 0.386 & 0.340 \\
$V_{c}(0 \mathrm{~K})(\mathrm{eV})^{\mathrm{a}, \mathrm{c}}$ & 0.685 & 1.519 \\
$V_{c}(300 \mathrm{~K})(\mathrm{eV})^{\mathrm{a}, \mathrm{c}}$ & 0.621 & 1.428 \\
$V_{v}(\mathrm{eV})^{\mathrm{a}, \mathrm{c}}$ & 0.265 & 0.000 \\
$V_{c}+a_{c} \varepsilon_{h y}(0 \mathrm{~K})(\mathrm{eV})$ & 1.058 & 1.519 \\
$V_{c}+a_{c} \varepsilon_{h y}(300 \mathrm{~K})(\mathrm{eV})$ & 0.994 & 1.428 \\
$V_{v}+a_{v} \varepsilon_{h y}(\mathrm{eV})$ & 0.192 & 0.000 \\
$C_{11}^{\mathrm{a}}=11.9 \times 10^{10} \mathrm{~Pa}$ & & \\
$C_{12}{ }^{\mathrm{a}}=5.38 \times 10^{10} \mathrm{~Pa}$ & & \\
$C_{44}{ }^{\mathrm{a}}=5.95 \times 10^{10} \mathrm{~Pa}$ & & \\
$b_{a x}{ }^{\mathrm{a}}=-1.8(\mathrm{eV})$ & & \\
$\varepsilon_{r}^{\mathrm{a}}(\mathrm{InAs})=15.15$ & & \\
\hline \hline
\end{tabular}

${ }^{\mathrm{a}}$ From Ref. 24.

${ }^{\mathrm{b}}$ From Ref. 25.

${ }^{c}$ From Ref. 27.

${ }^{\mathrm{d}}$ From Ref. 26.

$$
\begin{aligned}
\chi\left(\mathbf{k}^{\prime \prime}\right)= & \frac{-i b_{z}}{k_{x}^{\prime \prime} k_{y}^{\prime \prime}} \sum_{j=1}^{4} \frac{(-1)^{j}}{\phi_{j}}\left[f_{2} \exp \left(i \phi_{j}\right)-f_{1} \exp \left(-i \phi_{j}\right)\right. \\
& \left.-\frac{\left(f_{2}-f_{1}\right)}{\phi_{j}} \sin \left(\phi_{j}\right)\right] \exp \left[i \frac{\theta_{j}}{2}(1+f)\right]
\end{aligned}
$$

where

$$
\begin{gathered}
\theta_{1}=k_{x}^{\prime \prime} b_{x}+k_{y}^{\prime \prime} b_{y}, \\
\theta_{2}=-k_{x}^{\prime \prime} b_{x}+k_{y}^{\prime \prime} b_{y}, \\
\theta_{3}=-k_{x}^{\prime \prime} b_{x}-k_{y}^{\prime \prime} b_{y}, \\
\theta_{4}=k_{x}^{\prime \prime} b_{x}-k_{y}^{\prime \prime} b_{y}, \\
\phi_{j}=k_{z}^{\prime \prime} b_{z}-\theta_{j} \frac{(1-f)}{2} .
\end{gathered}
$$

We assume that energy gaps, band offsets, and carrier inverse effective masses vary linearly with In composition in strained $\mathrm{In}_{x} \mathrm{Ga}_{1-x}$ As inclusions in GaAs. The Fourier transforms of all terms in the electron and heavy-hole Hamiltonian equations can then be determined analytically, enabling a straightforward evaluation of each of the matrix elements in Eq. (2.1).

We derive the electron and hole envelope function Hamiltonian equations from three-band $\mathbf{k} \cdot \mathbf{P}$ theory. ${ }^{18,19}$ The electron Hamiltonian is given as

$$
\begin{aligned}
\hat{H}_{c}(\mathbf{r})= & -\frac{\hbar^{2}}{2 m_{0}} \nabla \frac{1}{m^{*}(\mathbf{r})} \nabla+V_{c}(\mathbf{r})+a_{c} \varepsilon_{h y}(\mathbf{r}) \\
& +d_{p z}(\mathbf{r})+e F z
\end{aligned}
$$

where $m^{*}(\mathbf{r})$ is the electron effective mass, $V_{c}(\mathbf{r})$ the unstrained conduction-band edge, $a_{c} \varepsilon_{h y}(\mathbf{r})$ the hydrostatic deformation of the conduction band edge, and $d_{p z}(\mathbf{r})$ the piezoelectric potential. The strain-induced band deformation causes the effective-mass parameters to vary from their bulk values. The variation of the electron effective mass with the (hydrostatically) strained band gap, $E_{g s}$, is given from threeband $\mathbf{k} \cdot \mathbf{P}$ theory as

$$
\frac{1}{m^{*}(\mathbf{r})}=1+\frac{2 E_{p}(\mathbf{r})}{3 E_{g s}(\mathbf{r})}+\frac{E_{p}(\mathbf{r})}{3\left[E_{g s}(\mathbf{r})+\triangle_{0}(\mathbf{r})\right]}+\delta(\mathbf{r}),
$$

where $E_{p}(\mathbf{r})$ is the Kane interband energy parameter, and $\delta(\mathbf{r})$ takes into account the contribution of remote bands to the conduction-band effective mass.

For the case of isotropic elastic constants, finding the analytic form of $\bar{H}_{\mathbf{k} \cdot \mathbf{k}^{\prime}}$ is a trivial matter for all but the piezoelectric term in $\hat{H}_{c}(\mathbf{r})$. In order to evaluate this term, using a similar technique to that employed in Ref. 17 we Fourier transform the Green's function for the piezoelectric potential presented in Ref. 20 and apply convolution theory, to obtain an analytic expression for the Fourier transform of the piezoelectric potential $\left(\mathbf{k}^{\prime \prime} \neq 0\right)$

$$
\begin{aligned}
\widetilde{d}_{p z}\left(\mathbf{k}^{\prime \prime}\right)= & \frac{-18 i \varepsilon_{0}}{\epsilon_{0} \sqrt{2 \pi}}\left(\frac{C_{11}+2 C_{12}}{C_{11}}\right)\left[\frac{e_{14}^{\text {bar }}}{\epsilon_{r}^{\text {bar }}} \chi\left(\mathbf{k}^{\prime \prime}\right) \frac{k_{x}^{\prime \prime} k_{y}^{\prime \prime} k_{z}^{\prime \prime}}{\left|\mathbf{k}^{\prime \prime}\right|^{4}}\right. \\
& \left.+\left(\frac{e_{14}^{\text {dot }}}{\boldsymbol{\epsilon}_{r}^{\text {dot }}}-\frac{e_{14}^{\text {bar }}}{\epsilon_{r}^{\text {bar }}}\right) \sum_{\mathbf{k}^{\prime \prime \prime}} \chi\left(\mathbf{k}^{\prime \prime \prime}-\mathbf{k}^{\prime \prime}\right) \chi\left(\mathbf{k}^{\prime \prime}\right) \frac{k_{x}^{\prime \prime} k_{y}^{\prime \prime} k_{z}^{\prime \prime}}{\left|\mathbf{k}^{\prime \prime}\right|^{4}}\right],
\end{aligned}
$$

where $\epsilon_{0}, \epsilon_{r}^{d o t}$, and $\epsilon_{r}^{b a r}$ are the permittivity of free space and relative permittivity of the dot and barrier material, and $e_{14}^{d o t}$ and $e_{14}^{\text {bar }}$ are the piezoelectric constants. In the diagonal (one-band) approximation used here, heavy holes have a large effective mass in the growth direction and a small effective mass in the growth plane, ${ }^{21}$ so that the dominant contribution to the heavy-hole kinetic energy is directed parallel to the $x-y$ plane. This contrasts with the light holes, where the dominant kinetic-energy term is directed parallel to the $z$ axis. From experiment, Stranski-Krastanow quantum dots have a large base-to-height ratio, i.e, $L_{x}=L_{y} \gg L_{z}$. The kinetic energy in the $x-y$ plane is thus reduced relative to that in the growth direction, leading to stronger heavy-hole confinement and weaker light-hole confinement. In addition, axial strain tends to reduce the light-hole confinement potential through most of the dot. ${ }^{5,17}$ The one-band valence Hamiltonian we use for the hole ground state is then given as

$$
\begin{aligned}
\hat{H}_{v}(\mathbf{r})= & \frac{\hbar^{2}}{2 m_{0}}\left\{\nabla_{\|}\left[\gamma_{1}(\mathbf{r})+\gamma_{2}(\mathbf{r})\right] \nabla_{\|}\right. \\
& \left.+\nabla_{z}\left[\gamma_{1}(\mathbf{r})-2 \gamma_{2}(\mathbf{r})\right] \nabla_{z}\right\} \\
& +V_{v}(\mathbf{r})+a_{v} \varepsilon_{h y}(\mathbf{r})-b_{a x} \varepsilon_{a x}(\mathbf{r}) \\
& +d_{p z}(\mathbf{r})+e F z
\end{aligned}
$$



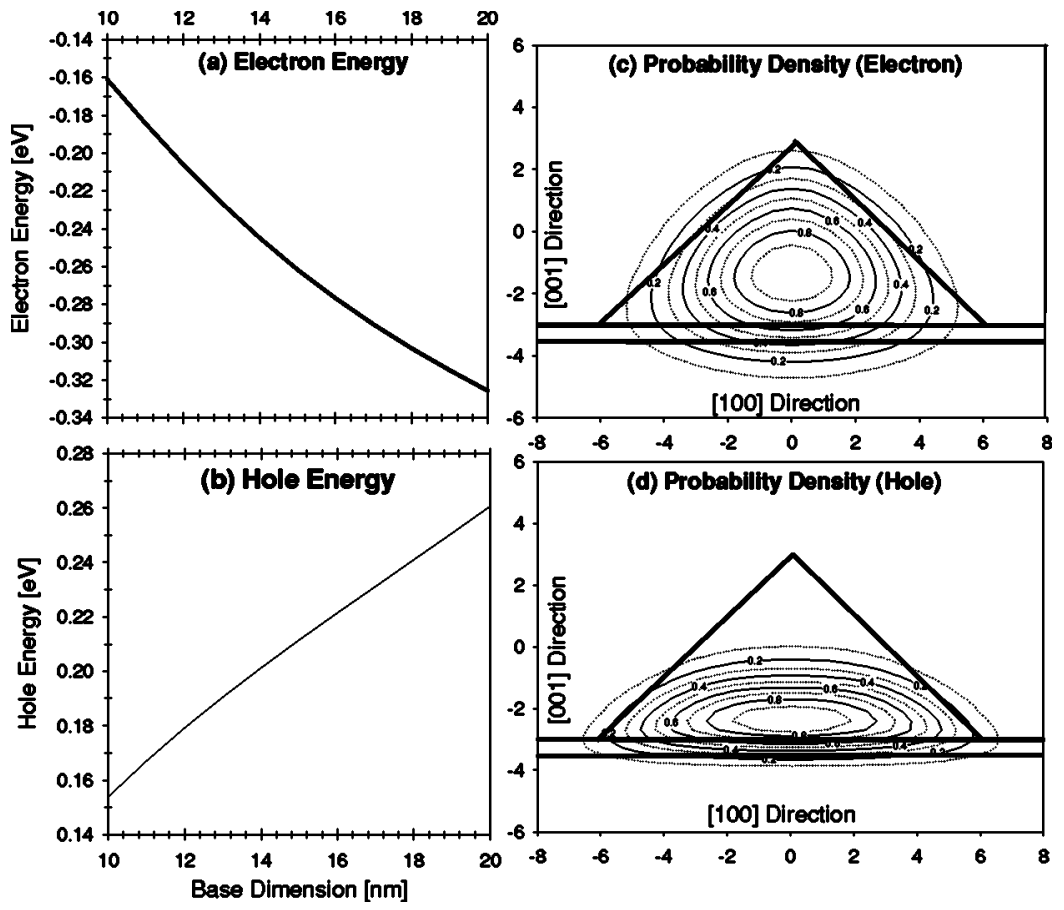

FIG. 1. Ground-state electron (a) and hole (b) confinement energies $(0 \mathrm{~K})$ as a function of dot size for InAs quantum pyramids of base:height ratio $2: 1$, on a $1-\mathrm{ml}$ InAs wetting layer (left). Probability density (arbitrary units) for a groundstate electron (c) and hole (d) in a pyramid of base $12 \mathrm{~nm}$ and height $6 \mathrm{~nm}$ on 1-ML wetting layer (right). where $V_{v}(\mathbf{r})$ is the unstrained valence band-edge energy, $a_{v} \varepsilon_{h y}(\mathbf{r})$ the hydrostatic deformation of the valence-band edge, and $-b_{a x} \varepsilon_{a x}(\mathbf{r})$ the axial-strain-induced shift in the heavy-hole band edge. In order to correctly describe the oneband Luttinger parameters $\gamma_{i}(\mathbf{r})$, we first relate them to their eight-band counterparts, $\gamma_{i}^{\prime}(\mathbf{r})$. In the full eight-band Hamiltonian, the interactions between the conduction-band minimum and valence-band maximum are considered explicitly, with $\gamma_{i}^{\prime}(\mathbf{r})$ then due to interactions with remote bands, and assumed to be independent of the built-in strain and the lowest-energy gap. The eight-band $\gamma_{i}^{\prime}(\mathbf{r})$ values are related to the one-band values by 22,23

$$
\gamma_{1}^{\prime}(\mathbf{r})=\gamma_{1}(\mathbf{r})-\frac{E_{P}(\mathbf{r})}{3 E_{g}(\mathbf{r})}, \quad \gamma_{2}^{\prime}(\mathbf{r})=\gamma_{2}(\mathbf{r})-\frac{E_{P}(\mathbf{r})}{6 E_{g}(\mathbf{r})},
$$

where $E_{g}$ is the unstrained band gap. The energy gap changes in a strained material, so that the one-band Luttinger parameters $\gamma_{i}$ will also change in a strained inclusion, as

$$
\gamma_{1}(\mathbf{r})=\gamma_{1}^{\prime}(\mathbf{r})+\frac{E_{P}(\mathbf{r})}{3 E_{g s}(\mathbf{r})}, \quad \gamma_{2}(\mathbf{r})=\gamma_{2}^{\prime}(\mathbf{r})+\frac{E_{P}(\mathbf{r})}{6 E_{g s}(\mathbf{r})}
$$

The parameters used are taken from Refs. 24-26 and the band offsets from Krijn. ${ }^{27}$ These are tabulated in Table I. We calculated the parameter values for InAs in a GaAs matrix, and for unstrained GaAs, and then assumed a linear variation of all parameters with alloy composition. This assumption is consistent with the parameters compiled by $\mathrm{Krijn}^{27}$. Although the (unstrained) band gap of $\operatorname{In}_{x} \mathrm{Ga}_{1-x}$ As has a moderately large bowing parameter, the strain contribution to the band gap introduces a bandgap bowing in the opposite direction which almost exactly counteracts this. ${ }^{27}$ The calculations presented below were carried out assuming roomtemperature energy gaps and effective masses. The effective mass and Luttinger parameters quoted from Ref. 25 are $0-\mathrm{K}$ values. These were related to the $300-\mathrm{K}$ values by using room-temperature values for the strained energy gap in Eqs. (2.5) and (2.8). Finally, we assume the GaAs values for the elastic constants in the dot and surrounding matrix. We have justified this choice previously, ${ }^{17,28}$ based on Keyes' scaling rule for elastic constants. ${ }^{29,30}$

\section{RESULTS}

Figures 1(a) and 1(b) show the variation of the groundstate electron and hole confinement energies, calculated as a function of dot size for InAs pyramids of base to height ratio 2:1 on a 1-ML InAs wetting layer. The results are in good agreement with previous calculations. Using the effectivemass renormalization procedure, we find several boundelectron states. For the dot shape considered in Figs. 1(c) and 1(d), we find four distinct electron bound states within the dot. In Table II, we compare our calculated ground state confinement energies for a dot of base $12 \mathrm{~nm}$ and height 6 $\mathrm{nm}$, with previous calculations., ${ }^{3,6}$ The calculated electron confinement energies are in good agreement with previous eight-band $\mathbf{k} \cdot \mathbf{P}$ calculations, ${ }^{3}$ sitting midway between the

TABLE II. Ground-state electron and hole confinement energies $(\mathrm{eV})$ at $0 \mathrm{~K}$, for a dot of base $12 \mathrm{~nm}$ and height $6 \mathrm{~nm}$. Numbers in italics were taken from graphs in the relevant references.

\begin{tabular}{lcccc}
\hline \hline Band-structure parameters & Elastic model & W.L. & $E_{\text {elec }}$ & $E_{\text {hole }}$ \\
\hline From Table I & plane-wave & None & 0.179 & 0.154 \\
From Table I & plane-wave & $1 \mathrm{ml}$ & 0.206 & 0.179 \\
From Stier et al. (Ref. 3) & plane-wave & $1 \mathrm{ml}$ & 0.211 & 0.175 \\
Stier et al. (Ref. 3) & CM & $1 \mathrm{ml}$ & 0.203 & 0.155 \\
Stier et al. (Ref. 3) & VFF & $1 \mathrm{ml}$ & 0.220 & 0.161 \\
From Table I & plane-wave & $1.5 \mathrm{ml}$ & 0.219 & 0.196 \\
From Cusack et al. (Ref. 6) & plane-wave & $1.5 \mathrm{ml}$ & 0.217 & 0.214 \\
Cusack et al. (Ref. 6) & VFF & $1.5 \mathrm{ml}$ & 0.165 & 0.250 \\
\hline \hline
\end{tabular}


values calculated using two different force-field models to determine the strain distribution. Our values lie about 10 $\mathrm{meV}$ above the electron energies calculated using the valence force-field model, and about $10 \mathrm{meV}$ below those calculated using a continuum mechanical method. The hole groundstate energy in Fig. 1(b) is consistently about 10\% further from the valence-band edge than that calculated in Ref. 3. To investigate the cause of this difference, we have calculated the hole confinement energy using our model and the bandstructure parameters from Ref. 3. The resulting confined state energy is within $4 \mathrm{meV}$ of that obtained using our parameters. Most of the observed difference must therefore be due to factors other than band-structure parameters, of which the most important is likely to be the different force-field parameters used, and consequent differences in the biaxial strain distribution. The difference is unlikely to be due to the use of a one-band rather than eight-band Hamiltonian here. If we were to include mixing between heavy- and light-hole bands in our Hamiltonian, these should tend to increase the ground-state hole confinement, pushing the ground-state valence level further away from the GaAs valence-band edge, thereby increasing the difference between the two sets of results. Reference 5 found a significantly larger hole confinement energy, and smaller electron confinement energy than Ref. 3. This is almost certainly due in part to a differing treatment of the band offset. There are other important differences between the two calculations, including the choice of a 1.5 -ML wetting layer in Ref. 5 , as opposed to the $1-\mathrm{ML}$ wetting layer assumed in Ref. 3. This results in a $15-\mathrm{meV}$ shift in hole confinement for the pyramid (Table II). Reference 5 also employed a different treatment of the electron and hole effective masses of strained InAs, taking an average of the results of empirical pseudopotential and ab initio local-density calculations, and finding values of $\gamma_{1}=8.201$, $\gamma_{2}=3.253$, and $m^{*}=0.04$. These compare to the strainrenormalized one-band effective masses of $\gamma_{1}=10.601, \gamma_{2}$ $=3.836$, and $m^{*}=0.042$ used for the present work. While there is good agreement between the calculated electron effective masses, the differences in $\gamma_{1}$ and $\gamma_{2}$ lead to different hole effective masses. The largest difference is seen for the hole effective mass in the growth direction, which is estimated from the strain-renormalized $\mathbf{k} \cdot \mathbf{P}$ parameters to be 0.341 , whereas Ref. 5 estimated a significantly larger value of 0.590 . The different choice of effective mass results in a 20-meV shift in the hole confinement energy (Table II). The comparisons in Table II indicate the range of envelope function parameters currently used to model strained InAs/GaAs quantum-dot structures. This range was considered in further detail in Ref. 3, where it was concluded that the greatest uncertainty in the calculated confined-state energies is associated with the assumed band-offset values, with differing assumptions concerning the elastic constants used also playing a role. We shall present most of our results here assuming the band-structure parameters listed in Table I. Calculations are presented in Sec. IV, which show that reasonable variations in the assumed band offsets or strain distribution do not qualitatively affect the main conclusions of our work.

Figures 1(c) and 1(d) show contours of constant probability density, calculated for the ground-state electron and hole wave functions for a pyramidal dot of base $12 \mathrm{~nm}$ and height $6 \mathrm{~nm}$ on a 1-ML wetting layer. The electron wave function is

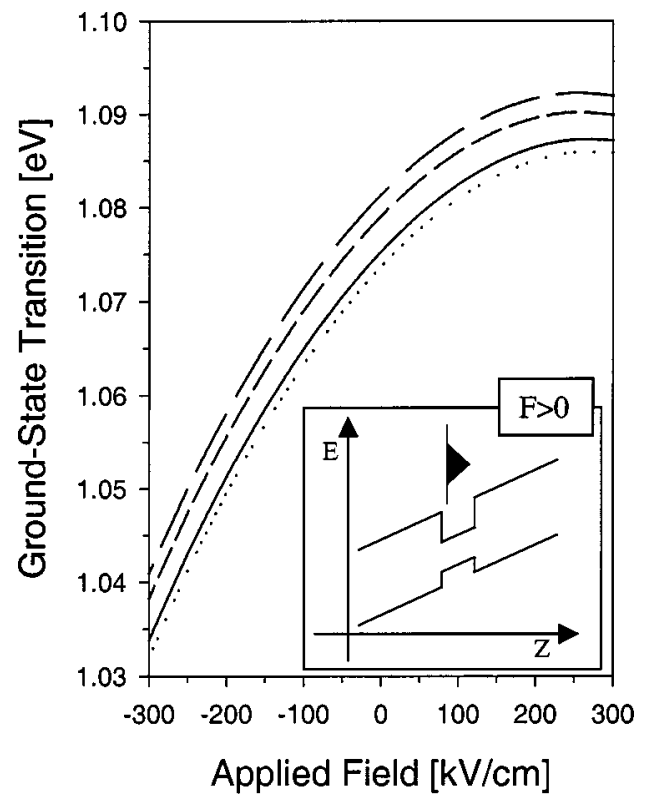

FIG. 2. Ground-state transition energy $(300 \mathrm{~K})$ as a function of applied field for pyramidal dots of base $12 \mathrm{~nm}$ and height $6 \mathrm{~nm}$ on a 1-ML wetting layer, using the piezoelectric potential of both dot and barrier material (solid line), not including the piezoelectric deformation (long-dashed line), and setting the piezoelectric deformation to that of the dot everywhere (short-dashed line) and that of the barrier everywhere (dotted line). Inset: variation of the band edge with position for $F>0$.

distributed relatively evenly throughout the pyramid, while the hole wave function is predominantly localized near the dot base as a result of the variation in the biaxial strain, $\varepsilon_{a x}=\varepsilon_{z z}-\left(\varepsilon_{x x}+\varepsilon_{y y}\right) / 2$, through the dot. Because the base of the dot is wider than the top, it has been shown ${ }^{2,6,17}$ that the base experiences a positive biaxial strain $\left(\varepsilon_{a x}>0\right)$, while the top is under negative biaxial strain $\left(\varepsilon_{a x}<0\right)$. This then leads through the term $-b_{a x} \varepsilon_{a x}$ to a deeper well for heavy holes at the base than at the top of the pyramid. The combination of the deeper well and large heavy-hole mass explains why the hole wave function is predominantly localized near the dot base. In contrast, the electron Hamiltonian [Eq. (2.4)] depends on strain only through the hydrostatic component $\varepsilon_{h y}$, whose magnitude is relatively constant through the dot. The hydrostatic strain $\varepsilon_{h y}$ is exactly constant in the model used here, where we assume isotropic elastic constants of equal magnitude in the dot and barrier. $\varepsilon_{h y}$ is no longer exactly constant when we use anisotropic elastic constants ${ }^{17}$ or different values of the elastic constants in the dot and barrier. ${ }^{3}$ Nevertheless, we expect, based on the above analysis, that the hole will lie below the ground state electron in any quantum dot of uniform composition whose shape tapers from a broad base to a narrow top. Figure 2 shows the calculated difference between the electron and hole ground state energies for the dot structure considered in Fig. 1 when an electric field is applied along the pyramid axis. For the present work, we follow the same convention as in Ref. 12, and have defined the applied field to be positive when it results in the conduction- and valence-band edges moving to higher energy above the dot and to lower energy below the dot, as illustrated in the inset to Fig. 2. Here, and for the remainder of this section, we use $300-\mathrm{K}$ parameters rather 
than $0-\mathrm{K}$ parameters as the room-temperature behavior of quantum-dot-based devices is of most practical long-term interest. Use of $300-\mathrm{K}$ parameters, rather than $0-\mathrm{K}$ parameters reduces the value of $E_{T R}(0)$, but has little effect on the calculated values of $\alpha$ and $\beta$. The solid line in Fig. 2 shows the transition including the piezoelectric potential; the longdashed line shows the transition with the piezoelectric potential set to zero, and the short-dashed and dotted lines show the transition using the dot and barrier parameters, respectively, for the piezoelectric potential everywhere. The close agreement between all of the above calculations occurs for two reasons: first, the piezoelectric potential is only significant near the corners of the pyramid ${ }^{20}$ away from the regions in which the electron and hole wave functions are concentrated. Second, the piezoelectric potential has odd symmetry about the (100) and (010) planes through the dot center, while the ground-state wave functions have even symmetry about these planes, so that the piezoelectric term does not shift the ground-state electron and hole energies in first-order perturbation theory. These two factors explain why the piezoelectric potential has little effect on the ground-state transition energy in (001)-grown, zinc-blende pyramidal dots. Setting the piezoelectric potential to the barrier value everywhere provides a good approximation of the full piezoelectric potential calculation. While this would at first seem surprising given the factor of 4 difference between $e_{14} / \varepsilon_{r}$ for the two materials, one should note that piezoelectric deformation occurs predominantly outside of the dot, so that most of the overlap between the piezoelectric fields and the charge-carrier wave functions will occur in the barrier material. For dots whose composition is graded from $\operatorname{In}_{x} \mathrm{Ga}_{1-x}$ As at the base to InAs at the top surface, there will be a smaller lattice mismatch between the dot and barrier material at the base of the dot, reducing the atomic displacement caused by the inclusion and hence weakening the piezoelectric field. As the pyramidal dot shape is truncated, the piezoelectric field becomes more concentrated at the corners of the dot while the electron and hole wave functions become more strongly localized toward the center of the dot. In both cases the piezoelectric shift in ground-state carrier confinement should be smaller than that seen for the pyramid. Test calculations have shown that the piezoelectric-induced shift in the ground-state transition energy is negligible in the graded, truncated pyramids considered below.

It can be seen from Fig. 2 that for moderate fields the transition energy varies quadratically with applied field, so that $E_{T R}(F)=E_{T R}(0)-\alpha F-\beta F^{2}$. The linear coefficient $\alpha$ depends directly on the initial separation of the electron and hole mean positions, i.e, on the built-in ground-state dipole moment of the dot. This is confirmed using second-order perturbation theory. The electron and hole Hamiltonian equations may be written in the presence of an applied field as

$$
\hat{H}=\hat{H}_{0}+\hat{H}^{\prime},
$$

where $\hat{H}_{0}$ is the unperturbed Hamiltonian of Eq. (2.4) or Eq. (2.6), and $\hat{H}^{\prime}=e F z$. The ground-state electron (hole) wave functions $\Psi_{e(h)}$ are given to first order in the applied electric field $F$ as

$$
\Psi_{e(h)}=\psi_{e(h)}^{0}+F \psi_{e(h)}^{\prime},
$$

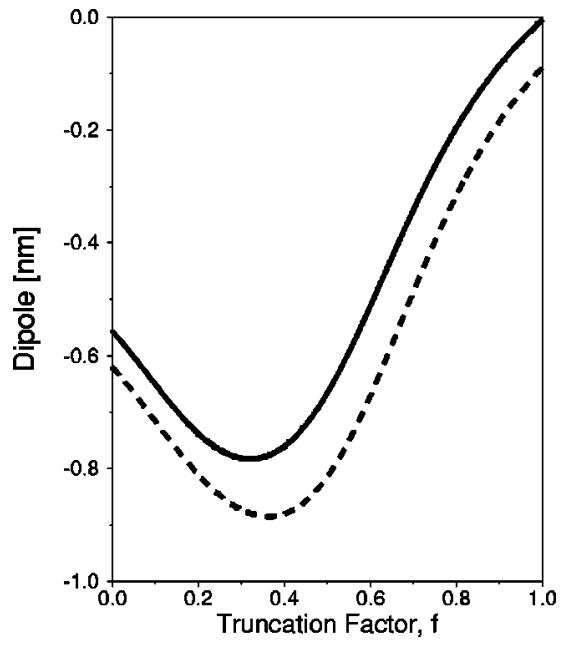

FIG. 3. Dipole $(300 \mathrm{~K})$ as a function of truncation factor $f$, at a constant dot height. [ $f=0$ for an ideal pyramid]. The solid line shows a truncated pyramid with base $18 \mathrm{~nm}$ and height $5.5 \mathrm{~nm}$. The dashed line shows a truncated pyramid with base $18 \mathrm{~nm}$ and height $4.935 \mathrm{~nm}$, sitting on a $1 \mathrm{ml}$ wetting layer so that the total height of dot plus wetting layer is $5.5 \mathrm{~nm}$.

where $\psi_{e(h)}^{0}$ is the ground-state wave function in zero field, and the first-order correction $F \psi_{e(h)}^{\prime}$ is written so as to emphasize that its magnitude varies linearly with $F$. From second-order perturbation theory, the ground-state electron energy $E_{e}$ then varies with applied field as

$$
E_{e}(F)=E_{e}(0)+e F\left\langle z_{e}\right\rangle+e F^{2}\left\langle\psi_{e}^{0}|z| \psi_{e}^{\prime}\right\rangle,
$$

where $\left\langle z_{e}\right\rangle=\left\langle\psi_{e}^{0}|z| \psi_{e}^{0}\right\rangle$ is the mean electron position along the pyramid axis. Using an equivalent expression for the hole energy we see that the transition energy, $E_{T R}$, varies to second order as

$$
\begin{aligned}
E_{T R}(F)= & E_{T R}(0)-e F\left(\left\langle z_{h}\right\rangle-\left\langle z_{e}\right\rangle\right) \\
& -e F^{2}\left(\left\langle\psi_{h}^{0}|z| \psi_{h}^{\prime}\right\rangle-\left\langle\psi_{e}^{0}|z| \psi_{e}^{\prime}\right\rangle\right) .
\end{aligned}
$$

The linear coefficient $\alpha$ is therefore identically equal to the ground-state dipole moment, $\alpha=e\left(\left\langle z_{h}\right\rangle-\left\langle z_{e}\right\rangle\right)$, while the quadratic term $\beta$ depends only on the polarizability of the ground-state electron wave function, $-\beta_{e} / e$ $=\left\langle\psi_{e}^{0}|z| \psi_{e}^{\prime}\right\rangle$, and of the ground-state hole wave function, $\beta_{h} / e=\left\langle\psi_{h}^{0}|z| \psi_{h}^{\prime}\right\rangle$.

The peak transition energy is seen at positive field in Fig. 2 , consistent with a negative dipole $d=\alpha / e$, with $d=-8.8$ $\AA$. By contrast, experimental Stark shift measurements both on InAs/GaAs (Ref. 12) and $\mathrm{In}_{x} \mathrm{Ga}_{1-x} \mathrm{As} / \mathrm{Al}_{x} \mathrm{Ga}_{1-x} \mathrm{As}$ QD structures ${ }^{13}$ show the peak transition energy at negative fields in the notation used here. The experimentally observed built-in dipole is therefore of opposite sign to that predicted from Fig. 2 and Eq. (3.1), with the hole-electron separation $d=4.0 \pm 1 \AA$ in Ref. 12. We therefore conclude that the structure of these buried Stranski-Krastinow-grown dots must be markedly different from an ideal constant composition pyramid, and now turn to consider what dot structures are consistent with the experimentally observed dipole and polarizability.

Figure 3 shows the calculated dipole in a truncated InAs 
pyramid of base width $B=18 \mathrm{~nm}$ and height $H=5.5 \mathrm{~nm}$ as a function of truncation factor, $f$. The value assumed for $H$ is similar to the value estimated from a TEM analysis of uncapped dots, and also to that inferred from the value of $\beta$ measured for capped dots. ${ }^{12} f$ represents the fraction of the total pyramid height removed so that, for example, a truncated pyramid with $f=0.75$ and $H=5.5 \mathrm{~nm}$ is formed by decapitating a full pyramid of height $22 \mathrm{~nm}$. The dashed line in the figure shows the dipole when a 1-ML InAs wetting layer is included, while the solid line shows the dipole with no wetting layer. The wetting layer deepens the hole potential near the base of the dot. The hole is always localized below the electron in this case, with $d=1 \AA$ even for a cuboidal dot $(f=1)$ sitting on the wetting layer. Moving from a cuboidal to a pyramidal geometry, the increasing anisotropy of the dot shape modifies the axial strain fields present in the dot to move the heavy holes away from the top surface of the dot and toward the dot base, so that the ground-state hole moves more rapidly toward the dot base than the ground-state electron, and the magnitude of the electron-hole separation increases. For the dot dimensions considered, the electron-hole separation reaches a maximum at $f \sim 0.35$, and then decreases again for smaller $f$. At $f$ $\sim 0.35$, the hole is already predominantly localized at the base of the dot. For smaller $f$, the increasingly pyramidal shape pushes the electron downwards more rapidly than the hole, so that the electron starts to "catch up" with the hole. It is clear that, regardless of the level of truncation, the mean electron position will always remain above that of the hole, up to the limit of a cuboidal dot $(f=1)$. When we set $f=1$, and assume no wetting layer, the electron and hole wave functions are both symmetric about the cuboid central plane, so that in this case $d=0$.

If the hole is to sit in the upper part of the dot, above the electron, we require a deeper heavy-hole potential at the top of the dot than at the base. Experimental studies generally indicate a dot shape tapering from the base to the top. The heavy-hole potential can nevertheless be deeper at the top than at the base if the dot is formed of an $\operatorname{In}_{x} \mathrm{Ga}_{1-x}$ As alloy, with the indium composition, $x$, increasing from the base to the top. We have assumed a linear composition gradient, but note that recent work ${ }^{11}$ suggests that the true composition profile may possibly be more complicated. Figure 4(a) shows the calculated electron-hole separation $d$ for dots with dimension $18 \times 5.5 \mathrm{~nm}^{2}$. The solid line shows a linearly graded cuboid, for which $d=0$ when $x=1$ at the base of the pyramid, with $d$ increasing to a maximum value of order $7 \AA$ near $x \sim 0.3$ at the base. A similar trend is observed for the truncated pyramid with $f=0.75$ (dashed line). The electron initially sits above the hole here, but the positions reverse with increasing grading. Figure 4(b) shows the calculated separations when the cuboid and truncated pyramid are sitting on a one monolayer $\operatorname{In}_{x} \mathrm{Ga}_{1-x}$ As wetting layer, with $x$ constant in the wetting layer and equal to the value at the dot base. For intermediate values of $x$, the wetting layer leads to a slight increase in $d$, as the electron is pulled down toward the wetting layer, but the hole remains pinned by the stronger axialstrain induced deformation at the base of the dot. Moving to large $x$, the heavy-hole confining potential becomes stronger in the well than the dot, so that the hole is pulled more
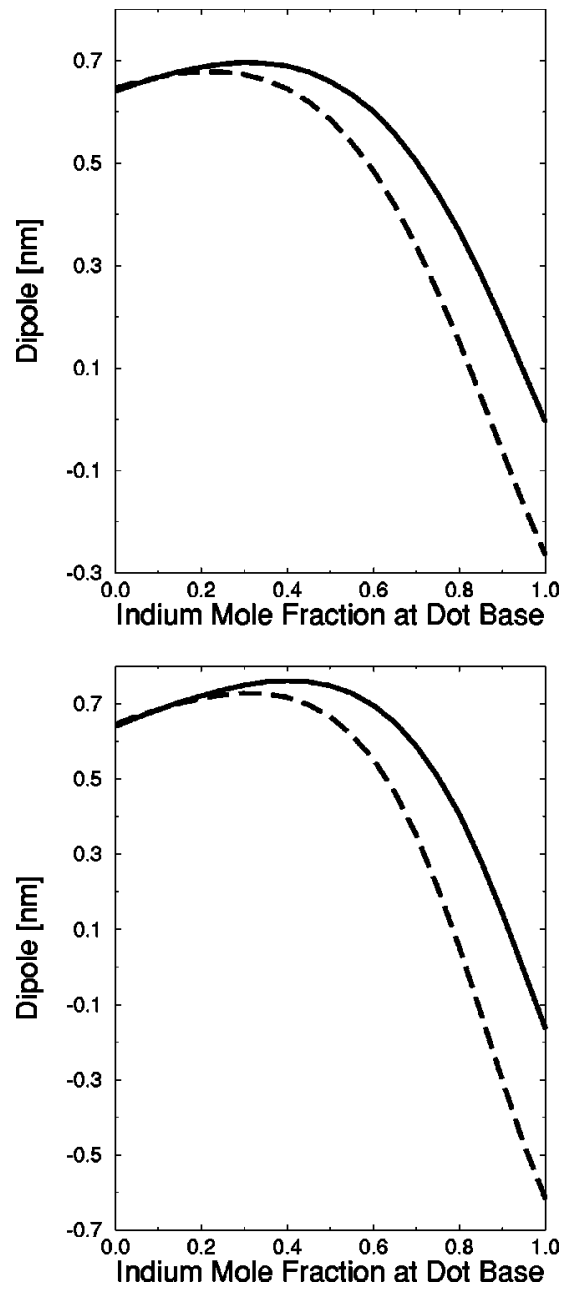

FIG. 4. Dipole (300 K) as a function of composition gradient for dots of base $18 \mathrm{~nm}$ and height $5.5 \mathrm{~nm}$, graded from $\operatorname{In}_{x} \mathrm{Ga}_{1-x} \mathrm{As}$ at the base to InAs at the top surface, (a) for dots with no wetting layer, and (b) for dots sitting on a 1-ML wetting layer. The solid lines are results for dots with a cuboidal geometry $(f=1)$, and the dashed lines are results for truncated dots with $f=0.75$.

strongly toward the wetting layer than the electron, shifting the dipole toward more negative values.

A fit to the experimentally observed electron-hole separation of $d=4 \AA$ can then be obtained for a variety of different dot geometries. We see from Fig. 4 that such a separation can be obtained, e.g., for graded composition cuboids and truncated pyramids both with and without a wetting layer, although the grading must be stronger in the presence of the wetting layer. Indeed a fit to the experimental separation may be obtained for disklike dots, dome-shaped dots, etc. The Stark shift data do not determine the precise detail of the dot shape, but do require severe compositional grading. The electron-hole separation will vary in graded truncated pyramids, both as a function of the dot grading $(x)$ and the truncation factor $f$. This is illustrated in Fig. 5, which plots contours of constant electron-hole separation as a function of grading (horizontal axis) and truncation factor (vertical axis) for dots with a base width of $18 \mathrm{~nm}$ and height $5.5 \mathrm{~nm}$, without a wetting layer. Interestingly, the data in Fig. 5 exclude pyramidal dots. Even with $100 \%$ linear grading from base to apex and no wetting layer, the electron still remains above the hole, giving a dipole of the wrong sign. 


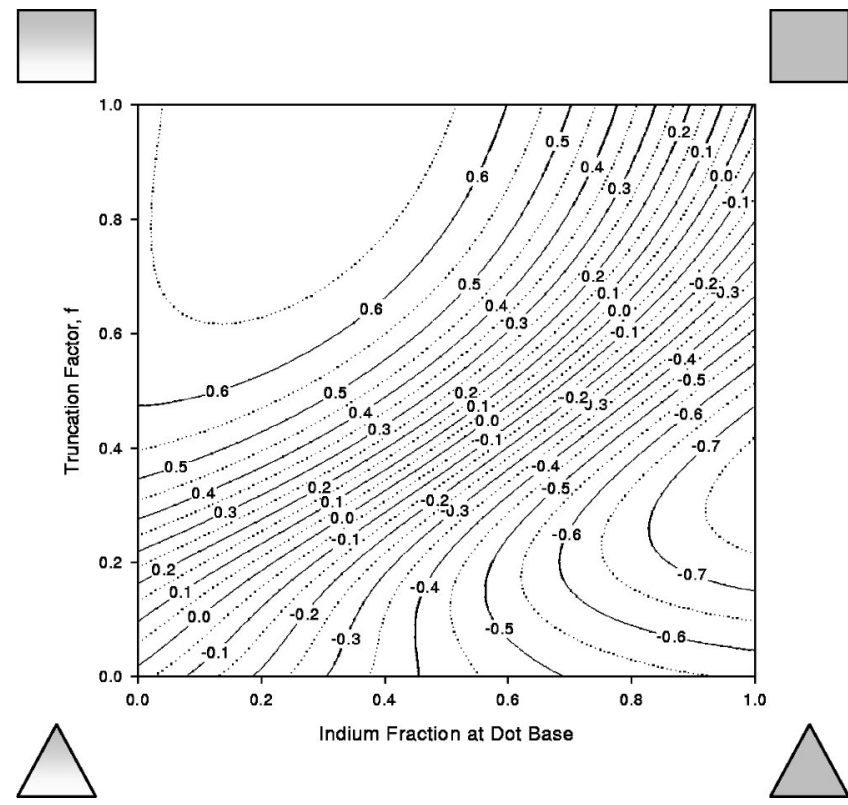

FIG. 5. Relative vertical position (in $\mathrm{nm}$ ) of holes and electrons $(300 \mathrm{~K})$ as a function of truncation and composition gradient, for dots of base $18 \mathrm{~nm}$ and (truncated) height $5.5 \mathrm{~nm}$.

The measured polarizability $\beta$ of self-organized quantum $\operatorname{dots}^{12}$ also provides information regarding their structure, and in particular their estimated height $H$. The polarizability of electrons and holes was widely studied in quantum-well structures. ${ }^{19,31}$ It can be shown for a particle of mass $m^{*}$ in an infinitely deep quantum well of width $H$ that the polarizability $\beta \propto m^{*} H^{4}$, which can also be written as $\beta \propto H^{2} /\left(E_{1}\right.$ $-E_{0}$ ), where $E_{1}-E_{0}$ is the energy separation between the first excited and ground-state levels. The polarizability therefore increases strongly with the quantum-well width. Most quantum-well studies have focused on wider quantum wells ( $H>\sim 7 \mathrm{~nm}$ ), for which the heavy-hole contribution is considerably larger than the electron contribution to the total polarizability, because $m_{h h}^{*} \gg m_{c}^{*}$.

Figure 6(a) shows the calculated electron and hole polarizabilities $\beta_{e}$ and $-\beta_{h}$ as functions of height $H$ for a range of different test structures. These include a graded truncated pyramid with base $18 \mathrm{~nm}, x=0.5$ and $f=0.75$ (solid line), an InAs cuboid with base $18 \mathrm{~nm}$ (dashed line), and an InAs quantum well (dotted line), each embedded in a GaAs matrix. The calculated polarizabilities follow the broad trend predicted by the infinite well analysis, with both $\beta_{e}$ and $\beta_{h}$ increasing with $H$ in all cases, and with $\beta_{h}$ about three times larger than $\beta_{e}$ in the widest $(7 \mathrm{~nm})$ quantum well and cuboid. The magnitudes of $\beta_{e}$ and $\beta_{h}$ become comparable in narrower structures, and the calculated values of $\beta_{e}$ exceed those of $\beta_{h}$ for $H \sim 3 \mathrm{~nm}$. This occurs because the small vertical dimension of the well restricts the motion of electrons and holes within the well, so that the effects of barrier penetration become significant. This is confirmed by Fig. 6(b), which plots $|\psi(z)|^{2}$, the integral of the probability density function over the $x-y$ plane, for several model structures, where
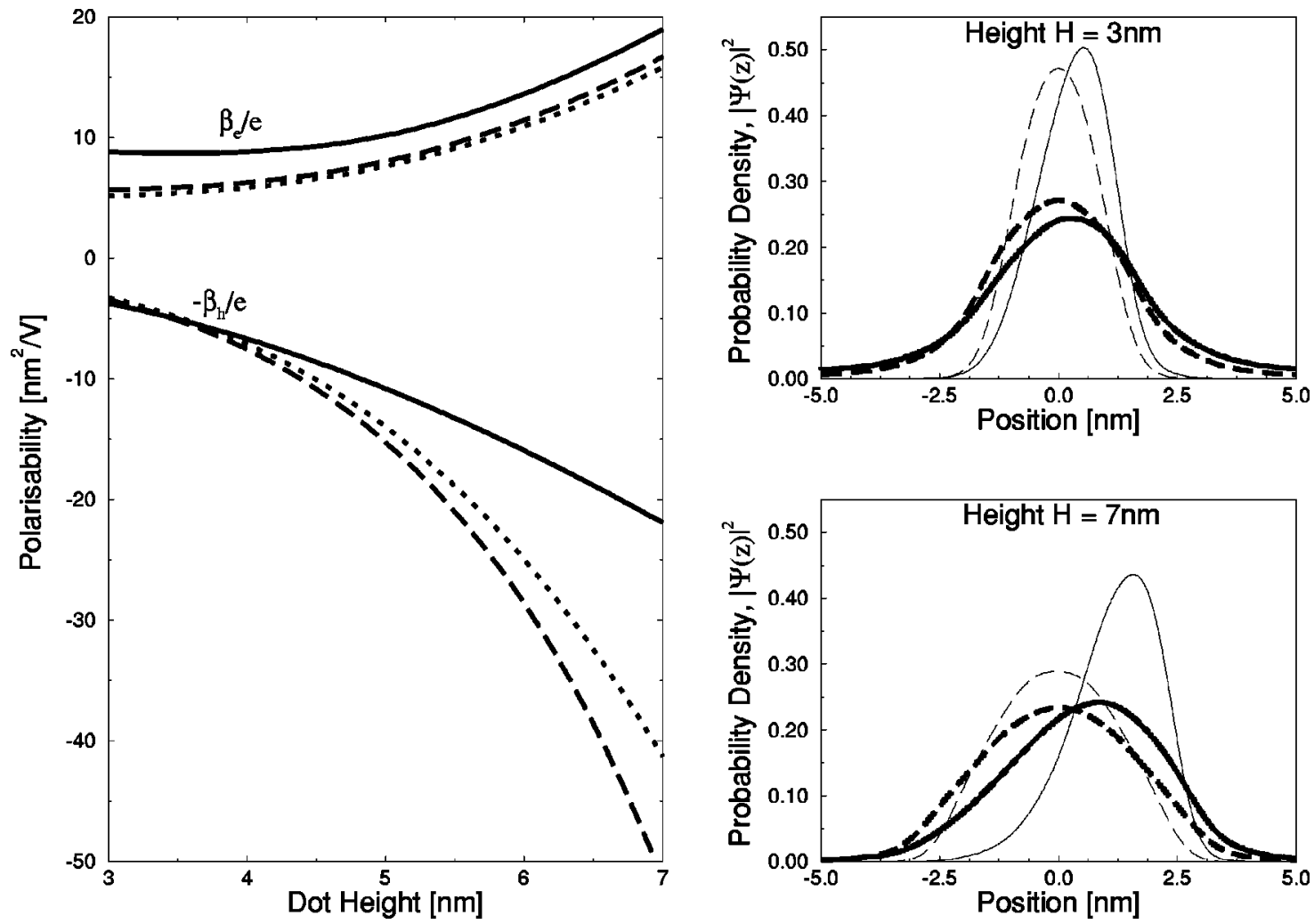

FIG. 6. (a) Polarizability (300 K), for electrons (top left) and holes (bottom left), as a function of height for dots of base $18 \mathrm{~nm}$. The solid lines are results for a graded truncated pyramid, with $f=0.75$ and $x=0.5$; the dashed lines are for a cuboid; and the dotted lines are for a quantum well. (b) $|\psi(z)|^{2}=\int_{-L_{x}}^{L_{x}} d x \int_{-L_{y}}^{L_{y}} d y|\psi(\mathbf{r})|^{2}$, the integral of the probability density function over the $x-y$ plane, for quantum dots of base $18 \mathrm{~nm}$, with heights of $3 \mathrm{~nm}$ (top right) and height $7 \mathrm{~nm}$ (bottom right). The thick lines are results for electrons, and the thin lines are results for holes. The solid lines are results for graded truncated dots with $x=0.5$ and 0.75 , and the dashed lines are results for cuboidal dots. 


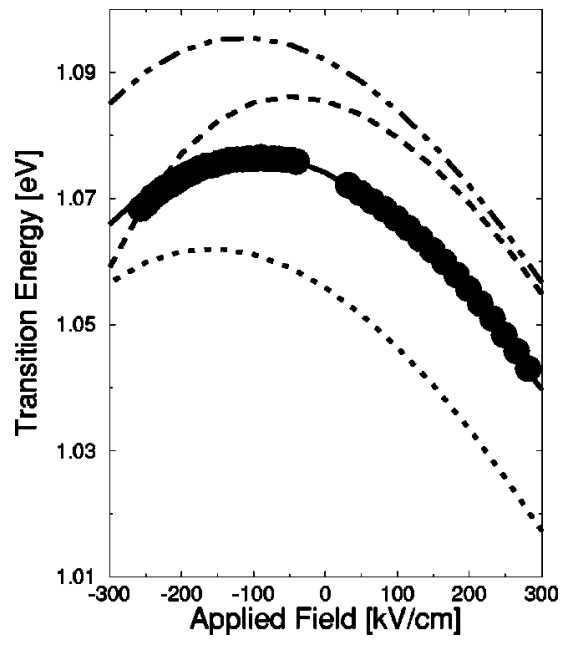

FIG. 7. Solid line: Fit to experimentally measured transition energy $(300 \mathrm{~K})$, for a graded truncated pyramid with base $18 \mathrm{~nm}$, height $5.5 \mathrm{~nm}$, and $f=0.81$, and graded linearly from $x=0.6$ at the base to $x=1.0$ at the top. The fit to experiment differs slightly from that quoted by the authors in Ref. 8, due to a slightly different treatment of the valence effective mass and elastic parameters. Also shown are the calculated transition energies for: a 100-meV upward shift of the InAs conduction and valence-band edges (dotted line); a 100-meV downward shift in these band edges (dashed line); and leaving band offsets unchanged, but using anisotropic elastic constants (double-dot-dashed line).

$$
|\psi(z)|^{2}=\int_{-L_{x}}^{L_{x}} d x \int_{-L_{y}}^{L_{y}} d y|\psi(\mathbf{r})|^{2} .
$$

We consider a graded truncated pyramid with base $18 \mathrm{~nm}$, $x=0.5$ and $f=0.25$ (solid line), and an InAs cuboid with base $18 \mathrm{~nm}$ (dashed line). The thick lines show the electron probability density, and the thin lines show the hole probability density. It can be seen for $H=3 \mathrm{~nm}$ that the small electron effective mass leads to a greater penetration of the electron than the hole into the GaAs barrier, increasing the effective size $H_{\text {eff }}$ of the electron state, and hence increasing the electron polarizability relative to the hole polarizability. This also explains why the electron polarizability is largest in the graded truncated pyramid, where the lower confinement energy leads to increased barrier penetration compared to the cuboid, and hence larger, $\beta_{e}$. By contrast, the hole polarizability $\beta_{h}$ is reduced in the graded truncated pyramid compared to the constant composition cuboid and the quantum well, reflecting the increased vertical localization of the hole wave function due to the variation in the zero-field heavyhole potential along the axis of the dot.

Finally we note that based on the above analysis we can obtain a very good fit to the experimentally observed Stark shift in InAs/GaAs quantum-dot structures (Fig. 7, solid line). ${ }^{12}$ The fit is obtained assuming a truncated pyramid with base $18 \mathrm{~nm}$, height $H=5.5 \mathrm{~nm}$, and truncation factor $f$ $=0.81$, and the dot graded linearly from $x=0.6$ at the base to $x=1.0$ at the top. This is slightly different to the dot shape which we presented previously, ${ }^{12}$ where $x=0.5$ at the dot base. This is due to minor changes in the way we treat the valence effective mass and elastic parameters here. While the dot structure chosen is by no means a unique fit to the data, its overall dimensions ( $B$ and $H$ ) are in good agreement with a structural analysis of uncapped dots formed under identical growth conditions. ${ }^{12}$ For this geometry, we observe electron states at five energy levels bound within the dot, two of which are singly degenerate, and three of which are (nominally) doubly degenerate. The calculated energy levels are fairly evenly spaced, with a separation between the ground state and first excited state of $62 \mathrm{meV}$.

\section{UNCERTAINTY ANALYSIS}

The one-band electron and hole Hamiltonian equations used here involve several parameters whose absolute values are still uncertain. It was shown in Ref. 3 that the values assumed for the conduction- and valence-band offsets are perhaps the most significant factor affecting the calculated electron and hole confined-state energies. We have therefore recalculated the variation of transition energy with electric field, $E_{T R}(F)$, for the dot structure of Fig. 7, including rigid upward and downward shifts of $0.1 \mathrm{eV}$ in the InAs bandedge energies. The results are indicated by the dotted and dashed lines, respectively, in Fig. 7. For an upward shift in the electron and hole band offsets (dotted line), we see a greater asymmetry in the Stark shift of the transition energy. This is because the shift in the band offset leads to a deeper potential well for holes and a shallower well for electrons, increasing the effects of the confining potential on the shape of the hole wave function, and reducing the asymmetry in the shape of the electron wave function. This would indicate that either a weaker compositional grading within the dot, or a more tapered dot geometry, would be required to obtain an improved fit in this case. The converse effect is seen for a downward shift in the electron and hole band offsets (dashed line), which would then require either stronger compositional grading, or a less tapered geometry to fit experiment.

We have omitted the influence of the exciton binding energy when calculating the interband transition energy. Two questions need to be addressed concerning this omission: first, what is the typical magnitude of this binding energy; and second, how does it vary with electric field, and thereby modify the calculated $\alpha$ and $\beta$ values? The ground-state exciton binding energy is determined by the Coulomb interaction between the ground-state electron and hole states, as described in the Appendix, and can be determined numerically by carrying out an appropriate sum over the plane-wave coefficients for the wave functions, and the Fourier transform of the Coulomb interaction. ${ }^{34}$ We choose instead to take an analytical approach here, in order to derive general conclusions regarding the influence of the exciton binding energy, $J_{e h}$. We see from Fig. 6 that the electron and hole probability density distributions are close to Gaussians. The binding energy $J_{e h}$ can then be determined analytically for several cases, as described in the Appendix. For the electron, we estimate that $L_{e}=2.86 \mathrm{~nm}$ in the growth direction and $B_{e}$ $=5.55 \mathrm{~nm}$ in the growth plane. For the hole, $L_{h}=1.58 \mathrm{~nm}$ and $B_{h}=5.17 \mathrm{~nm}$. For simplicity, we take the relative permittivity to be the InAs value here. When the electron and hole sit on top of each other, with no built-in dipole, the exciton binding energy is then estimated from Eq. (A5) as $17.7 \mathrm{meV}$. This value is comparable to previous calculations of the exciton binding energy in dots of similar 
dimension, ${ }^{32,33}$ and with that obtained for pyramidal dots of similar base dimension. ${ }^{3}$ When the electron and hole are displaced vertically to create a dipole of length $d$, the binding energy initially decreases quadratically with $d$, as confirmed in Fig. 2 of Ref. 33. We estimate from Eq. (A9) that, for small $d, J_{e h}$ varies as $J_{e h}(d)=\left(17.7-0.324 d^{2}\right)\left(\mathrm{nm}^{2}\right) \mathrm{meV}$ for the $B$ and $L$ values listed above. For the dot shape of Fig. 7 , the built-in dipole $d=\left\langle z_{h}\right\rangle-\left\langle z_{e}\right\rangle$ changes by approximately $1 \AA$ for every $24-\mathrm{kV} / \mathrm{cm}$ change in applied electric field $F$. Based on this analysis, inclusion of the exciton binding energy and its field dependence would therefore shift the calculated curve in Fig. 7 downward by $\sim 20 \mathrm{meV}$, and reduce the calculated polarizability by about $3 \%$. Preliminary numerical calculations indicate that this analytical model underestimates the variation of $J_{e h}(d)$ but that, nevertheless, the effects of the exciton binding energy can be accounted for by slightly increasing the dot height, and decreasing the radius of the dot compared to the value of the fit in Fig. 7.

The one-band model should be appropriate to describe the electron confinement energies and wave functions, but may introduce a systematic error into the calculated valence-band ground-state energies and wave functions, as we ignore mixing effects with the light-hole bands. The ground-state wave function in the pyramidal dot of base width $13.6 \mathrm{~nm}$ considered in Ref. 3 had less than $10 \%$ light-hole character, ${ }^{35}$ so that a considerable vertical displacement of the light-hole center of mass with respect to the heavy-hole center of mass would be required to significantly modify the average ground-state hole position. This is not the case. Both heavyand light-hole components of the ground-state hole level lie toward the base of the dot for this dot shape. ${ }^{35}$ We thus expect that use of a full valence-band Hamiltonian might alter the precise detail of the calculated dipole moments and polarizabilities, but should not significantly change the overall conclusions of this work.

The other approximation made here which needs further consideration is the assumption of isotropic elastic constants. It has been shown ${ }^{17}$ that, since the QD shape has a lower symmetry than that of the elastic parameters, the asymmetry of the dot shape dominates in determining the strain distribution, so that the isotropic and anisotropic models should give similar results. However, whereas $\varepsilon_{h y}$ is exactly constant within the dot for the case of isotropic elastic constants, $\varepsilon_{h y}$ is observed to vary with position when anisotropic cubic elastic constants are used, with $\varepsilon_{h y}$ increasing approximately linearly along the central axis of a constant composition pyramid. ${ }^{3,17}$ This will give an additional linear variation of the conduction- and valence-band edge energies along the dot axis. We have therefore calculated the electron and hole wave functions for the anisotropic case, replacing the isotropic strain tensor of Eq. (2.3) with the anisotropic, cubic, strain tensor from Ref. 17. For the pyramidal dot structure considered in Fig. 1(b), the position of the hole wave function is predominantly determined by the axial strain distribution, which maintains the same shape for both isotropic and anisotropic models, so that the position of the hole is unaffected by the introduction of cubic anisotropy. The electron wave function experiences a shift of $1 \AA$ toward the base of the dot, as hydrostatic strain introduces a potential gradient toward the dot base. This shift in electron position is an order of magnitude too small to reverse the sign of the zero-field dipole. Considering the graded truncated dot shape of Fig. 7, the shift in the hole position is again relatively small, and the electron position shifts $\sim 0.3 \AA$ toward the base of the dot. The Stark shift calculated using anisotropic elastic constants is plotted as the double-dot dashed line in Fig. 7. There is a blueshift of about $20 \mathrm{meV}$ compared to the isotropic case, and the peak position is not significantly different from the results for the isotropic model. In this case, a better fit to experiment could be obtained with a slightly larger dot base dimension. We conclude that the use of anisotropic elastic constants would also not significantly modify our results.

\section{CONCLUSION}

We have used one-band electron and hole Hamiltonian equations to investigate the different factors influencing the magnitude and sign of the built-in dipole in strained $\mathrm{In}_{x} \mathrm{Ga}_{1-x} \mathrm{As}$ quantum-dot structures, and also investigated the polarizability of the dots. We have demonstrated that the built-in strain will always lead to the hole center of mass lying below the electron center of mass in a constant composition dot whose cross-sectional area tapers from the base to the top. The inclusion of an InAs wetting layer further increases the magnitude of the electron-hole dipole. The calculated dipole moment is of opposite sign to that determined from recent quantum-confined Stark effect experimental measurements. ${ }^{12,13} \mathrm{We}$ show that the correct sign and magnitude of dipole can be obtained if we include composition grading, with the indium composition increasing from the base to the top of the tapered dot. This conclusion is also consistent with the results of recent grazing-incidence diffraction measurements on uncapped dots. ${ }^{10}$ The theoretical analysis presented here, combined with relevant experimental data, has provided microstructural information which must be taken into account in future studies of self-organized quantum-dot structures. Our results demonstrate conclusively that the measured Stark effect in self-organized quantum-dot structures cannot be explained by assuming a pyramidal dot shape.

\section{ACKNOWLEDGMENTS}

The authors would like to thank Igor Itskevich, Maurice Skolnick, David Mowbray, and Paul Fry for much useful discussion on Stark shifts, and also James Downes, Gary Pearson, and Aleksey Andreev for useful discussions on strain. We are grateful to Oliver Stier for kindly providing band-mixing data for the dot shape considered in Ref. 3, and acknowledge the Engineering and Physical Sciences Research Council (U.K.) for providing financial support to J.A.B.

\section{APPENDIX: EXCITON BINDING ENERGY}

When the ground-state electron and hole levels in a quantum dot are sufficiently localized that their extent is shorter than the bulk exciton effective Bohr radius, the ground-state exciton binding energy is then given to a good approximation by 


$$
J_{e h}=\frac{e^{2}}{4 \pi \varepsilon_{0} \varepsilon_{r}} \int_{-\infty}^{\infty} d \mathbf{r}_{e} \int_{-\infty}^{\infty} d \mathbf{r}_{h} \frac{\left|\Psi_{e}\left(\mathbf{r}_{e}\right)\right|^{2}\left|\Psi_{h}\left(\mathbf{r}_{h}\right)\right|^{2}}{\left|\mathbf{r}_{h}-\mathbf{r}_{e}\right|} .
$$

The six-dimensional integral in Eq. (A1) must, in general, be solved numerically in order to determine the value of $J_{e h}$. For the plane-wave basis functions used in this paper, this involves carrying out an appropriate sum over the plane wave coefficients and the Fourier transform of the Coulomb interaction. ${ }^{34}$ Instead, we choose here to analyze the variation of $J_{e h}$ with dot size and applied electric field by approximating the electron and hole probability density functions by Gaussian functions, for which case integral (A1) can be solved analytically. ${ }^{36}$ This approximation is shown in Fig. 6 to be a reasonable assumption. In this case, the electron and hole probability densities are given, respectively, by

$$
\begin{aligned}
& \left|\Psi_{e}\left(\mathbf{r}_{e}\right)\right|^{2}=\frac{1}{\pi^{3 / 2} L_{e} B_{e}^{2}} \exp \left[-\frac{\left(z_{e}+d / 2\right)^{2}}{L_{e}^{2}}-\frac{\rho_{e}^{2}}{B_{e}^{2}}\right], \\
& \left|\Psi_{h}\left(\mathbf{r}_{h}\right)\right|^{2}=\frac{1}{\pi^{3 / 2} L_{h} B_{h}^{2}} \exp \left[-\frac{\left(z_{h}-d / 2\right)^{2}}{L_{h}^{2}}-\frac{\rho_{h}^{2}}{B_{h}^{2}}\right],
\end{aligned}
$$

where $L_{e}$ and $L_{h}$ describe the extent of the electron and hole localization along the growth $(z)$ direction, $B_{e}$ and $B_{h}$ describe their in-plane extent, and the electron and hole are centered a distance $d$ apart. It can be shown that the value of the integral (A1) is unchanged when we replace $L_{e}$ and $L_{h}$ and $B_{e}$ and $B_{h}$ by

$$
L=\sqrt{\frac{L_{e}^{2}+L_{h}^{2}}{2}}, \quad B=\sqrt{\frac{B_{e}^{2}+B_{h}^{2}}{2}},
$$

respectively. We solve Eq. (A1) by making the change of variables $\mathbf{u}=\left(\mathbf{r}_{e}-\mathbf{r}_{h}\right)$ and $\mathbf{v}=\left(\mathbf{r}_{e}+\mathbf{r}_{h}\right)$. We can then find analytical expressions for $J_{e h}$ in several limiting cases.

(i) $L=B, d=0$ :

$$
J_{e h}=\frac{e^{2}}{(2 \pi)^{3 / 2} \varepsilon_{0} \varepsilon_{r}} \frac{1}{L} .
$$

(ii) $L \neq B, d=0$ :

$$
J_{e h}=\frac{e^{2}}{(2 \pi)^{3 / 2} \varepsilon_{0} \varepsilon_{r}} \frac{1}{\sqrt{B^{2}-L^{2}}} \tan ^{-1}\left[\frac{\sqrt{B^{2}-L^{2}}}{L}\right] .
$$

This reduces to Eq. (A4) when $L=B$, and to the value previously derived by Warburton et al. ${ }^{36}$ for the twodimensional $(L=0)$ case.

(iii) $L=B, d \neq 0$ :

$$
J_{e h}=\frac{e^{2}}{(2 \pi)^{3 / 2} \varepsilon_{0} \varepsilon_{r}} \frac{\sqrt{\pi}}{d \sqrt{2}} \operatorname{erf}\left[\frac{d}{L \sqrt{2}}\right],
$$

which, for small $d / L$, reduces to

$$
J_{e h}=\frac{e^{2}}{(2 \pi)^{3 / 2} \varepsilon_{0} \varepsilon_{r}} \frac{1}{L}\left[1-\frac{d^{2}}{6 L^{2}}\right] .
$$

(iv) $L=0, d \neq 0$ :

$$
J_{e h}=\frac{e^{2}}{(2 \pi)^{3 / 2} \varepsilon_{0} \varepsilon_{r}} \frac{\pi}{2 B} \exp \left[\frac{d^{2}}{2 B^{2}}\right] \operatorname{erfc}\left[\frac{d}{2 B}\right] .
$$

(v) $L \neq B, d \neq 0$. We find for small $d / L$ that

$$
\begin{aligned}
J_{e h}= & E_{0}-\frac{d^{2}}{2}\left[\frac{E_{0}}{L^{2}}-\frac{e^{2}}{(2 \pi)^{3 / 2} \varepsilon_{0} \varepsilon_{r}} \frac{1}{L^{2}\left(B^{2}-L^{2}\right)}\right. \\
& \left.\times\left(\frac{B^{2}}{\sqrt{B^{2}-L^{2}}} \tan ^{-1}\left[\frac{\sqrt{B^{2}-L^{2}}}{L}\right]-L\right)\right],
\end{aligned}
$$

where $E_{0}$ is equivalent to $J_{e h}$ in Eq. (A5)
${ }^{1}$ D. Bimberg, M. Grundmann, and N. N. Ledentsov, Quantum Dot Heterostructures (Wiley, New York, 1998).

${ }^{2}$ M. Grundmann, O. Stier, and D. Bimberg, Phys. Rev. B 52, 11969 (1995).

${ }^{3}$ O. Stier, M. Grundmann, and D. Bimberg, Phys. Rev. B 59, 5688 (1999).

${ }^{4}$ A. J. Williamson and A. Zunger, Phys. Rev. B 59, 15819 (1999).

${ }^{5}$ M.A. Cusack, P.R. Briddon, and M. Jaros, Phys. Rev. B 54, R2300 (1996).

${ }^{6}$ M. A. Cusack, P. R. Briddon, and M. Jaros, Physica B 253, 10 (1998).

${ }^{7}$ K. Chang and J.-B. Xia, Solid State Commun. 104, 351 (1997).

${ }^{8}$ N. N. Ledentsov, V. A. Shchukin, M. Grundmann, N. Kirstaedterr, J. Böhrer, O. Schmidt, D. Bimberg, V. M. Ustinov, A. Yu. Egorov, A. E. Zhukov, P. S. Kop'ev, S. V. Zaitsev, N. Yu. Gordeev, Zh. I. Alferov, A. I. Borokov, A. O. Kosogov, S. S. Ruvimov, P. Werner, U. Gösele, and J. Heydenreich, Phys. Rev. B 54, 8743 (1996).

${ }^{9}$ J. M. Moison, F. Houzay, F. Barthe, L. Leprince, E. André, and
O. Vatel, Appl. Phys. Lett. 64, 196 (1994).

${ }^{10}$ I. Kegel, T. H. Metzger, P. Fratzl, J. Peisl, A. Lorke, J. M. Garcia, and P. M. Petroff, Europhys. Lett. 45, 222 (1999).

${ }^{11}$ N. Liu, J. Tersoff, O. Baklenov, A. L. Holmes, Jr., and C. K. Shih, Phys. Rev. Lett. 84, 334 (2000).

${ }^{12}$ P. W. Fry, I. E. Itskevich, D. J. Mowbray, M. S. Skolnick, J. A. Barker, E. P. O'Reilly, L. R. Wilson, I. A. Larkin, P. A. Maksym, M. Hopkinson, M. Al-Khafaji, J. P. R. David, A. G. Cullis, G. Hill, and J. C. Clark, Phys. Rev. Lett. 84, 733 (2000).

${ }^{13}$ S. Raymond, J. P. Reynolds, J. L. Merz, S. Fafard, Y. Feng, and S. Charbonneau, Phys. Rev. B 58, R13 415 (1998).

${ }^{14}$ B. Wang, Y. Peng, F. Zhao, W. Chen, S. Liu, and C. Gao, J. Cryst. Growth 186, 43 (1998).

${ }^{15}$ S. Raymond, S. Fafard, P. J. Poole, A. Wojs, P. Hawrylak, S. Charbonneau, D. Leonard, R. Leon, P. M. Petroff, and J. L. Merz, Phys. Rev. B 54, 11548 (1996).

${ }^{16}$ T. Benabbas, P. Francois, Y. Androussi, and A. Lefebvre, J. Appl. Phys. 80, 2763 (1996).

${ }^{17}$ A. D. Andreev, J. R. Downes, D. A. Faux, and E. P. O'Reilly, J. Appl. Phys. 86, 297 (1999). 
${ }^{18}$ J. A. Barker and E. P. O'Reilly, Physica E 4, 231 (1999).

${ }^{19}$ G. Bastard, Wave Mechanics Applied to Semiconductor Heterostructures (Halstead, New York, 1988).

${ }^{20}$ J. H. Davies, J. Appl. Phys. 84, 1358 (1998).

${ }^{21}$ E. P. O'Reilly and A. Ghiti, in Quantum Well Lasers, edited by P. S. Zory (Academic Press, New York, 1993), p. 329.

${ }^{22}$ C. R. Pidgeon and R. N. Brown, Phys. Rev. 146, 575 (1966).

${ }^{23}$ A. T. Meney, B. Gonul, and E. P. O'Reilly, Phys. Rev. B 50, 10893 (1994).

${ }^{24}$ Numerical Data and Functional Relationships in Science and Technology, edited by O. Madelung, Landolt-Börnstein New Series, Group III, Vol. 17a (Springer-Verlag, Berlin, 1982).

${ }^{25}$ P. Lawaetz, Phys. Rev. B 4, 3460 (1971).

${ }^{26}$ S. Adachi, Physical Properties of III/V Semiconductor Compounds (Wiley, New York, 1992).

${ }^{27}$ M. P. M. C. Krijn, Semicond. Sci. Technol. 6, 27 (1991).

${ }^{28}$ J. R. Downes, D. A. Faux, and E. P. O’Reilly, J. Appl. Phys. 81, 6700 (1997).
${ }^{29}$ R. W. Keyes, J. Appl. Phys. 33, 3371 (1962).

${ }^{30}$ A. D. Prins and D. J. Dunstan, Philos. Mag. B 58, 37 (1988).

${ }^{31}$ M. Silver, P. D. Greene, and A. R. Adams, Appl. Phys. Lett. 67, 2904 (1995)

${ }^{32} \mathrm{Ph}$. Lelong and G. Bastard, in Proceedings of the 23rd International Conference on the Physics of Semiconductors, edited by M. Scheffler and R. Zimmerman (World Scientific, Singapore, 1996), p. 1377.

${ }^{33}$ N. Susa, IEEE J. Quantum Electron. 32, 1760 (1996).

${ }^{34}$ A. D. Andreev and E. P. O'Reilly, in Excitonic Processes in Condensed Matter, edited by R. T. Williams and W. M. Yen, Electrochemical Society Proceedings Series, Vol. 98-25 (Pennington, NJ, 1998), p. 271.

${ }^{35}$ O. Stier (private communication).

${ }^{36}$ R. J. Warburton, B. T. Miller, C. S. Dürr, C. Bödefeld, K. Karrai, J. P. Kotthaus, G. Medeiros-Ribeiro, P. M. Petroff, and S. Huant, Phys. Rev. B 58, 16221 (1998). 